\title{
Synthesis of Strained Macrocyclic Biaryls for Enthalpy-Driven Ring-Opening
}

\section{Polymerization}

Howard M. Colquhoun, ${ }^{* \dagger}$ Zhixue Zhu, ${ }^{\dagger}$ Christopher C. Dudman, ${ }^{\ddagger}$ Caroline A. O'Mahoney, ${ }^{\S}$ David J. Williams $^{\S}$ and Michael G. B. Drew ${ }^{\dagger}$

Department of Chemistry, University of Reading, Whiteknights, Reading, UK RG6 6AD,

Imperial Chemical Industries plc, The Heath, Runcorn, Cheshire, WA7 4QE

$\S$ Department of Chemistry, Imperial College, South Kensington, London, UK SW7 2AY

\section{SYNTHESIS AND CHARACTERIZATION OF LINEAR PRECURSOR-OLIGOMERS}

General procedure for nucleophilic aromatic substitution (precursors 1 and 4). The synthesis of 1 is taken as an example. A mixture of 4-chloro-4'-hydroxybenzophenone (23.45 g, 0.1 mole), 4,4'(hexafluoroisopropylidene)diphenol (13.45 g, 0.04 mole), anhydrous potassium carbonate $(8.29 \mathrm{~g}, 0.06$ mol), DMAc $(250 \mathrm{~mL})$ and toluene $(100 \mathrm{~mL})$ was heated at reflux under nitrogen, with Dean-Stark distillation of water. After $3.5 \mathrm{~h}$ the toluene was distilled off and the temperature was raised to $160{ }^{\circ} \mathrm{C}$. After a further $4 \mathrm{~h}$ the reaction mixture was cooled to room temperature, poured into water $(1000 \mathrm{~mL})$ and the precipitate was filtered off. After washing with water and then methanol, the solid was dried at $80{ }^{\circ} \mathrm{C}$ for $3 \mathrm{~h}$ and purified by chromatography (DCM) to give a white solid. A similar procedure was used for the preparation of $\mathbf{4}$.

4,4'-Bis[4"'-(4'"'-chlorobenzoylphenoxy)]hexafluoroisopropylidenediphenyl (1). Yield 80\%; m.p. $171{ }^{\circ} \mathrm{C} ;{ }^{1} \mathrm{H} \mathrm{NMR}\left(\mathrm{CH}_{3} \mathrm{SO}_{3} \mathrm{H} / \mathrm{CD}_{2} \mathrm{Cl}_{2} 1: 5,250 \mathrm{MHz}\right) \delta(\mathrm{ppm}) 8.10(\mathrm{~d}, \mathrm{~J}=9.1 \mathrm{~Hz}, 4 \mathrm{H}), 7.91(\mathrm{~d}, \mathrm{~J}=8.8$ $\mathrm{Hz}, 4 \mathrm{H}), 7.73(\mathrm{~d}, \mathrm{~J}=8.8 \mathrm{~Hz}, 4 \mathrm{H}), 7.59(\mathrm{~d}, \mathrm{~J}=9.1 \mathrm{~Hz}, 4 \mathrm{H}), 7.29(\mathrm{~d}, \mathrm{~J}=9.1 \mathrm{~Hz}, 4 \mathrm{H}), 7.28(\mathrm{~d}, \mathrm{~J}=9.1 \mathrm{~Hz}$, $4 \mathrm{H}) ;{ }^{13} \mathrm{C} \mathrm{NMR}\left(\mathrm{CH}_{3} \mathrm{SO}_{3} \mathrm{H} / \mathrm{CD}_{2} \mathrm{Cl}_{2} 1: 5,62.5 \mathrm{MHz}\right) \delta(\mathrm{ppm}) 202.4,167.9,155.2,145.4,139.0,135.3$, 
133.3, 131.4, 130.8, 126.8, 121.4, 119.2; IR (Nujol) $1652 \mathrm{~cm}^{-1}$ (vCO); MS (FAB) calcd. for

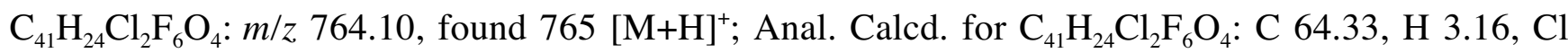
9.26. Found: $\mathrm{C}$ 64.21, H 3.20, $\mathrm{Cl} 9.49 \%$. Crystal data for 1: $\mathrm{C}_{41} \mathrm{H}_{24} \mathrm{C}_{12} \mathrm{~F}_{6} \mathrm{O}_{4}, M=765.5$, monoclinic,

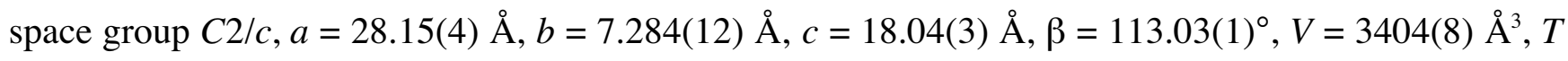
$=293 \mathrm{~K}, Z=4, D_{\mathrm{c}}=1.494 \mathrm{~g} \mathrm{~cm}^{-3}, \mu(\mathrm{Mo}-\mathrm{K} \alpha)=0.270 \mathrm{~mm}^{-1}, F(000)=1560 ; 3264$ independent reflections, $R_{1}=0.085, w R_{2}=0.203$ for 2559 independent observed reflections $\left[2 \theta \leq 52^{\circ}, I>2 \sigma(I)\right]$.

3,6-Bis-(4'-(4'"-chlorobenzoyl)phenoxy)xanthone (4). Yield 81.2\%; m.p. $227{ }^{\circ} \mathrm{C} ;{ }^{1} \mathrm{H}$ NMR $\left(\mathrm{CDCl}_{3} / \mathrm{TFA}, 250 \mathrm{MHz}\right) \delta(\mathrm{ppm}) 8.40\left(\mathrm{~d}, \mathrm{~J}=9.0 \mathrm{~Hz}, 2 \mathrm{H}_{\mathrm{g}}\right), 7.93(\mathrm{~d}, \mathrm{~J}=8.7 \mathrm{~Hz}, 4 \mathrm{H}), 7.79(\mathrm{~d}, \mathrm{~J}=8.5 \mathrm{~Hz}$, 4H), $7.55(\mathrm{~d}, \mathrm{~J}=8.5 \mathrm{~Hz}, 4 \mathrm{H}), 7.29(\mathrm{~d}, \mathrm{~J}=8.7 \mathrm{~Hz}, 4 \mathrm{H}), 7.24(\mathrm{dd}, \mathrm{J}=9.0,2.2 \mathrm{~Hz}, 2 \mathrm{H}), 7.13(\mathrm{~d}, \mathrm{~J}=2.2$ $\mathrm{Hz}, 2 \mathrm{H}) ;{ }^{13} \mathrm{C} \mathrm{NMR}\left(\mathrm{CH}_{3} \mathrm{SO}_{3} \mathrm{H} / \mathrm{CD}_{2} \mathrm{Cl}_{2} 1: 5,75 \mathrm{MHz}\right) \delta(\mathrm{ppm}) 214.6,199.3,174.7,167.6,160.6,158.6$, $141.3,134.3,133.8,132.7,129.6,129.5,121.2,119.6,111.0,105.3$; IR (Nujol) 1645 (vCO), 1269(vCO-C); MS (FAB) calcd. for $\mathrm{C}_{39} \mathrm{Cl}_{2} \mathrm{H}_{22} \mathrm{O}_{6}: m / z$ 656.08, found $657[\mathrm{M}+\mathrm{H}]^{+}$; Anal. Calcd. for $\mathrm{C}_{39} \mathrm{Cl}_{2} \mathrm{H}_{22} \mathrm{O}_{6}$ : C 71.24, H 3.37, Cl 10.78. Found: C 71.32, H 3.31, Cl 10.85\%.

General procedure for Friedel-Crafts acylation (precursors 2 and 3). The synthesis of $\mathbf{2}$ is taken as an example. Anhydrous aluminium chloride (16 g, $0.120 \mathrm{~mol}$ ) was suspended in 1,2dichlorobenzene $(150 \mathrm{~mL})$ under nitrogen, and 4-chlorobenzoyl chloride (12 g, $68 \mathrm{mmol})$ was added. To this solution was added 4,4'-diphenoxydiphenylsulfone (12 g, $30 \mathrm{mmol})$ and the mixture was heated at $40{ }^{\circ} \mathrm{C}$ for $2 \mathrm{~h}$, then at $100{ }^{\circ} \mathrm{C}$ for a further $2 \mathrm{~h}$. The mixture was then filtered and poured into icewater $(1000 \mathrm{~mL})$ containing concentrated hydrochloric acid $(45 \mathrm{~mL})$. The precipitate was recovered by filtration and washed with water, then dried at $80{ }^{\circ} \mathrm{C}$ overnight and recrystallised from toluene with charcoal treatment to yield a white solid. A similar procedure was used in the preparation of $\mathbf{3}$.

4,4'-Bis-(4"'-(4"'--chlorobenzoyl)phenoxy)diphenylsulfone (2). Yield 70\%; m.p. $220{ }^{\circ} \mathrm{C} ;{ }^{1} \mathrm{H}$ NMR $\left(\mathrm{CH}_{3} \mathrm{SO}_{3} \mathrm{H} / \mathrm{CD}_{2} \mathrm{Cl}_{2} 1: 5,300 \mathrm{MHz}\right) \delta(\mathrm{ppm}) 8.04(\mathrm{~d}, \mathrm{~J}=8.8 \mathrm{~Hz}, 4 \mathrm{H}), 7.96(\mathrm{~d}, \mathrm{~J}=8.8 \mathrm{~Hz}, 4 \mathrm{H}), 7.83(\mathrm{~d}, \mathrm{~J}$ $=8.6 \mathrm{~Hz}, 4 \mathrm{H}), 7.62(\mathrm{~d}, \mathrm{~J}=8.6 \mathrm{~Hz}, 4 \mathrm{H}), 7.30(\mathrm{~d}, \mathrm{~J}=8.8 \mathrm{~Hz}, 4 \mathrm{H}), 7.23(\mathrm{~d}, \mathrm{~J}=8.8 \mathrm{~Hz}, 4 \mathrm{H}) ;{ }^{13} \mathrm{C} \mathrm{NMR}$ 
$\left(\mathrm{CH}_{3} \mathrm{SO}_{3} \mathrm{H} / \mathrm{CD}_{2} \mathrm{Cl}_{2} 1: 5,75 \mathrm{MHz}\right) \delta(\mathrm{ppm}) 200.8,163.1,159.9,142.5,136.7,135.7,133.5,133.2,130.6$, 130.1, 129.8, 120.8, 119.4; IR (Nujol) $1646 \mathrm{~cm}^{-1}$ (vCO); MS (FAB) calcd. for $\mathrm{C}_{38} \mathrm{Cl}_{2} \mathrm{H}_{24} \mathrm{O}_{6} \mathrm{~S}, \mathrm{~m} / z$ 678.07; found $679[\mathrm{M}+\mathrm{H}]^{+}$; Anal. Calcd. for $\mathrm{C}_{38} \mathrm{Cl}_{2} \mathrm{H}_{24} \mathrm{O}_{6} \mathrm{~S}$ : C 67.16, H 3.56, Cl 10.43, S 4.71. Found: C 67.29, H 3.56, Cl 10.45, S 4.74\%.

2,8-Bis(4'-(4"'-chlorobenzoyl)phenoxy)dibenzofuran (3). Yield $76.6 \%$; m.p. $199{ }^{\circ} \mathrm{C} ;{ }^{1} \mathrm{H}$ NMR $\left(\mathrm{CH}_{3} \mathrm{SO}_{3} \mathrm{H} / \mathrm{CD}_{2} \mathrm{Cl}_{2} 1: 5,300 \mathrm{MHz}\right) \delta(\mathrm{ppm}) 7.99(\mathrm{~d}, \mathrm{~J}=8.9 \mathrm{~Hz}, 4 \mathrm{H}), 7.83(\mathrm{~d}, \mathrm{~J}=8.6 \mathrm{~Hz}, 4 \mathrm{H}), 7.77(\mathrm{~d}, \mathrm{~J}$ $=2.4 \mathrm{~Hz}, 2 \mathrm{H}), 7.73(\mathrm{~d}, \mathrm{~J}=8.9 \mathrm{~Hz}, 2 \mathrm{H}), 7.64(\mathrm{~d}, \mathrm{~J}=8.6 \mathrm{~Hz}, 4 \mathrm{H}), 7.35(\mathrm{dd}, \mathrm{J}=8.9$ and $2.4 \mathrm{~Hz}, 2 \mathrm{H}), 7.19$ $(\mathrm{d}, \mathrm{J}=8.9 \mathrm{~Hz}, 4 \mathrm{H}) ;{ }^{13} \mathrm{C} \mathrm{NMR}\left(\mathrm{CH}_{3} \mathrm{SO}_{3} \mathrm{H} / \mathrm{CD}_{2} \mathrm{Cl}_{2} 1: 5,75 \mathrm{MHz}\right) \delta(\mathrm{ppm}) 201.2,167.5,154.9,150.0$, 143.2, 137.1, 133.9, 132.3, 129.9, 127.1, 125.6, 121.4, 117.8, 113.8, 113.6; IR (Nujol) 1654 (vCO), 1248, $1167 \mathrm{~cm}^{-1}(v \mathrm{C}-\mathrm{O}-\mathrm{C})$; MS (FAB) calcd. for $\mathrm{C}_{38} \mathrm{Cl}_{2} \mathrm{H}_{22} \mathrm{O}_{5}: \mathrm{m} / z$ 628.08, found $629[\mathrm{M}+\mathrm{H}]^{+}$; Anal. Calcd. for $\mathrm{C}_{38} \mathrm{Cl}_{2} \mathrm{H}_{22} \mathrm{O}_{5}$ : C 72.51, $\mathrm{H} 3.52, \mathrm{Cl} 11.26$. Found: $\mathrm{C} 72.60, \mathrm{H} 3.44, \mathrm{Cl} 11.28 \%$.

General procedure for electrophilic sulfonylation (precursors 9, 10, 11). The synthesis of 10 is taken as an example. Anhydrous ferric chloride $(0.02 \mathrm{~g}, 1 \mathrm{mmol})$ was added to a stirred solution of 1,4diphenoxybenzene $(13.11 \mathrm{~g}, 50 \mathrm{mmol})$ and 4-chlorobenzenesulfonyl chloride $(29.5 \mathrm{~g}, 140 \mathrm{mmol})$ in trichlorobenzene $(15 \mathrm{~mL})$ at $120{ }^{\circ} \mathrm{C}$. The resulting mixture was heated at $150{ }^{\circ} \mathrm{C}$ for $24 \mathrm{~h}$ until no further $\mathrm{HCl}$ was evolved, cooled, and poured into methanol (400 mL) to give a brown precipitate. The precipitate was stirred in petroleum ether $\left(40-60{ }^{\circ} \mathrm{C}, 400 \mathrm{~mL}\right)$. The crude product was recrystallised from toluene $(100 \mathrm{~mL})$ with charcoal treatment to give a white crystalline solid. A similar procedure was applied for the preparation of 9 and $\mathbf{1 1 .}$

1,4-Bis(4'-(4"'-chlorobenzenesulfonyl)phenoxy)benzene (10).Yield 23\%; m.p. $267{ }^{\circ} \mathrm{C} ;{ }^{1} \mathrm{H}$ NMR $\left(\mathrm{DMSO}-d_{6}, 250 \mathrm{MHz}\right) \delta(\mathrm{ppm}) 7.96(\mathrm{~d}, \mathrm{~J}=8.9 \mathrm{~Hz}, 4 \mathrm{H}), 7.95(\mathrm{~d}, \mathrm{~J}=8.7 \mathrm{~Hz}, 4 \mathrm{H}), 7.69(\mathrm{~d}, \mathrm{~J}=8.7 \mathrm{~Hz}$, 4H), $7.22(\mathrm{~s}, 4 \mathrm{H}), 7.16(\mathrm{~d}, \mathrm{~J}=8.9 \mathrm{~Hz}, 4 \mathrm{H}) ;{ }^{13} \mathrm{C} \mathrm{NMR}\left(\mathrm{CDCl}_{3} / \mathrm{TFA}, 62.5 \mathrm{MHz}\right) \delta(\mathrm{ppm}) 163.2,152.0$, 141.2, 139.3, 133.6, 130.4, 130.3, 129.1, 122.7, 118.3; MS (CI) calcd. for $\mathrm{C}_{30} \mathrm{H}_{20} \mathrm{Cl}_{2} \mathrm{O}_{6} \mathrm{~S}_{2}: m / z$ 610.00, found 610 . 
4,4'-Bis(4"'-(4'"'-chlorobenzenesulfonyl)phenoxy)diphenylsulfone (11).Yield 66.9\%; amorphous;

${ }^{1} \mathrm{H}$ NMR $\left(\mathrm{DMSO}-d_{6}, 250 \mathrm{MHz}\right) \delta(\mathrm{ppm}) 8.01(\mathrm{~d}, \mathrm{~J}=8.8 \mathrm{~Hz}, 4 \mathrm{H}), 7.99(\mathrm{~d}, \mathrm{~J}=8.8 \mathrm{~Hz}, 4 \mathrm{H}), 7.98(\mathrm{~d}, \mathrm{~J}=$ $8.7 \mathrm{~Hz}, 4 \mathrm{H}), 7.71(\mathrm{~d}, \mathrm{~J}=8.7 \mathrm{~Hz}, 4 \mathrm{H}), 7.29(\mathrm{~d}, \mathrm{~J}=8.8 \mathrm{~Hz}, 4 \mathrm{H}+4 \mathrm{H}) ;{ }^{13} \mathrm{C} \mathrm{NMR}\left(\mathrm{CDCl}_{3}, 75 \mathrm{MHz}\right) \delta$ (ppm) 159.9, 159.7, 140.1, 140.0, 137.2, 136.8, 130.2, 130.1, 129.7, 129.0, 119.6; MS (FAB) calcd. for $\mathrm{C}_{36} \mathrm{H}_{24} \mathrm{Cl}_{2} \mathrm{O}_{8} \mathrm{~S}_{3}: m / z, 750.00$, found $751[\mathrm{M}+\mathrm{H}]^{+}$.

General procedure for nucleophilic aromatic substitution (precursor 12, 13, 14, 15 and 16). The synthesis affording 12 and $\mathbf{1 3}$ is taken as an example. A mixture of resorcinol (5.51 g, $50 \mathrm{mmol})$, 4,4'dichlorodiphenylsulfone $(31.59 \mathrm{~g}, 110 \mathrm{mmol})$, anhydrous potassium carbonate $(27.64 \mathrm{~g}, 200 \mathrm{mmol})$, DMAc $(250 \mathrm{~mL})$ and toluene $(100 \mathrm{~mL})$ was heated at reflux under nitrogen, with Dean-Stark distillation of water. After $3.5 \mathrm{~h}$ the toluene was distilled off and the reaction temperature was raised to $160{ }^{\circ} \mathrm{C}$. After a further $4 \mathrm{~h}$ the reaction mixture was cooled to room temperature, poured into water $(1000 \mathrm{~mL})$ and the precipitate was filtered off. After washing with water and then methanol, the solid was dried at $80^{\circ} \mathrm{C}$ for $3 \mathrm{~h}$ and purified by chromatography (DCM) to give white solid 12 and 13. A similar procedure was applied to the preparation of $\mathbf{1 4 , 1 5}$ and $\mathbf{1 6 .}$

1,3-Bis(4'-(4"'-chlorobenzenesulfonyl)phenoxy)benzene (12).yield 31.4\%; m.p. $198{ }^{\circ} \mathrm{C} ;{ }^{1} \mathrm{H}$ NMR $\left(\mathrm{CDCl}_{3}, 250 \mathrm{MHz}\right) \delta(\mathrm{ppm}) 7.88(\mathrm{~d}, \mathrm{~J}=8.8 \mathrm{~Hz}, 4 \mathrm{H}), 7.86(\mathrm{~d}, \mathrm{~J}=8.8 \mathrm{~Hz}, 4 \mathrm{H}), 7.48(\mathrm{~d}, \mathrm{~J}=8.8 \mathrm{~Hz}, 4 \mathrm{H})$, $7.39(\mathrm{t}, \mathrm{J}=8.2 \mathrm{~Hz}, 1 \mathrm{H}), 7.05(\mathrm{~d}, \mathrm{~J}=8.8 \mathrm{~Hz}, 4 \mathrm{H}), 6.88(\mathrm{dd}, \mathrm{J}=8.2$ and $2.3 \mathrm{~Hz}, 2 \mathrm{H}), 6.74(\mathrm{t}, \mathrm{J}=2.3 \mathrm{~Hz}$, $1 \mathrm{H}) ;{ }^{13} \mathrm{C} \mathrm{NMR}\left(\mathrm{CDCl}_{3}, 75 \mathrm{MHz}\right) \delta(\mathrm{ppm}) 161.4,156.5,140.4,139.8,135.5,131.3,130.1,129.6,129.0$, 118.3, 116.4, 112.2; MS (FAB) calcd. for $\mathrm{C}_{30} \mathrm{H}_{20} \mathrm{Cl}_{2} \mathrm{O}_{6} \mathrm{~S}_{2}: m / z 610.00$, found $611[\mathrm{M}+\mathrm{H}]^{+}$.

Precursor 13. Yield 13.1\%; m.p. $91{ }^{\circ} \mathrm{C} ;{ }^{1} \mathrm{H}$ NMR $\left(\mathrm{CDCl}_{3}, 250 \mathrm{MHz}\right) \delta(\mathrm{ppm}) 7.90(\mathrm{~d}, \mathrm{~J}=8.9 \mathrm{~Hz}$, $8 \mathrm{H}), 7.88(\mathrm{~d}, \mathrm{~J}=8.8 \mathrm{~Hz}, 4 \mathrm{H}), 7.49(\mathrm{~d}, \mathrm{~J}=8.8 \mathrm{~Hz}, 4 \mathrm{H}), 7.41(\mathrm{t}, \mathrm{J}=8.3 \mathrm{~Hz}, 2 \mathrm{H}), 7.08(\mathrm{~d}, \mathrm{~J}=8.9 \mathrm{~Hz}$, 4H), $7.06(\mathrm{~d}, \mathrm{~J}=8.9 \mathrm{~Hz}, 4 \mathrm{H}), 6.89(\mathrm{dd}, \mathrm{J}=8.3$ and $2.2 \mathrm{~Hz}, 4 \mathrm{H}), 6.75(\mathrm{t}, \mathrm{J}=2.2 \mathrm{~Hz}, 2 \mathrm{H}) ;{ }^{13} \mathrm{C} \mathrm{NMR}$ $\left(\mathrm{CDCl}_{3}, 62.5 \mathrm{MHz}\right) \delta(\mathrm{ppm}) 161.8,161.5,157.0,156.9,140.7,140.2,136.5,135.8,131.8,130.5,130.3$, 
130.1, 129.4, 118.7, 118.6, 116.8, 112.6; MS (FAB) calcd. for $\mathrm{C}_{48} \mathrm{H}_{32} \mathrm{Cl}_{2} \mathrm{O}_{10} \mathrm{~S}_{3}: \mathrm{m} / z$ 934.05, found 935 $[\mathrm{M}+\mathrm{H}]^{+}$.

Precursor 14. Yield 20.7\%; m.p. $210{ }^{\circ} \mathrm{C} ;{ }^{1} \mathrm{H} \mathrm{NMR}\left(\mathrm{CDCl}_{3}, 250 \mathrm{MHz}\right) \delta(\mathrm{ppm}) 8.04(\mathrm{~d}, \mathrm{~J}=8.7 \mathrm{~Hz}$, 2H), $8.03(\mathrm{~d}, \mathrm{~J}=8.7 \mathrm{~Hz}, 2 \mathrm{H}), 7.99(\mathrm{~d}, \mathrm{~J}=9.0 \mathrm{~Hz}, 2 \mathrm{H}), 7.94(\mathrm{~d}, \mathrm{~J}=9.0 \mathrm{~Hz}, 2 \mathrm{H}), 7.93(\mathrm{~d}, \mathrm{~J}=8.8 \mathrm{~Hz}$, 2H), $7.89(\mathrm{~d}, \mathrm{~J}=8.8 \mathrm{~Hz}, 2 \mathrm{H}), 7.70(\mathrm{~d}, \mathrm{~J}=8.7 \mathrm{~Hz}, 2 \mathrm{H}+2 \mathrm{H}), 7.52(\mathrm{~d}, \mathrm{~J}=8.8 \mathrm{~Hz}, 2 \mathrm{H}), 7.51(\mathrm{~d}, \mathrm{~J}=8.8$ $\mathrm{Hz}, 2 \mathrm{H}), 7.12(\mathrm{~d}, \mathrm{~J}=9.0 \mathrm{~Hz}, 2 \mathrm{H}), 7.11(\mathrm{~d}, \mathrm{~J}=9.0 \mathrm{~Hz}, 2 \mathrm{H}) ;{ }^{13} \mathrm{C} \mathrm{NMR}\left(\mathrm{CDCl}_{3}, 62.5 \mathrm{MHz}\right) \delta(\mathrm{ppm})$ $160.3,160.2,144.6,144.4,142.0,141.6,140.6,140.5,140.2,137.3,137.2,130.7,130.6,130.2,130.1$, 129.6, 129.5, 128.8, 128.7, 120.0; MS (CI) calcd. for $\mathrm{C}_{36} \mathrm{H}_{24} \mathrm{Cl}_{2} \mathrm{O}_{7} \mathrm{~S}_{3}: m / z$ 734.01, found $735[\mathrm{M}+\mathrm{H}]^{+}$.

3,6-Bis(4'-(4"'-chlorobenzenesulfonyl)phenoxy)xanthone (15). Yield 8.6\%; m.p. $211{ }^{\circ} \mathrm{C} ;{ }^{1} \mathrm{H}$ NMR $\left(\mathrm{CDCl}_{3}, 250 \mathrm{MHz}\right) \delta(\mathrm{ppm}) 8.34(\mathrm{~d}, \mathrm{~J}=8.7 \mathrm{~Hz}, 2 \mathrm{H}), 7.98(\mathrm{~d}, \mathrm{~J}=9.0 \mathrm{~Hz}, 4 \mathrm{H}), 7.91(\mathrm{~d}, \mathrm{~J}=8.8 \mathrm{~Hz}, 4 \mathrm{H})$, $7.52(\mathrm{~d}, \mathrm{~J}=8.8 \mathrm{~Hz}, 4 \mathrm{H}), 7.20(\mathrm{~d}, \mathrm{~J}=9.0 \mathrm{~Hz}, 4 \mathrm{H}), 7.05(\mathrm{dd}, \mathrm{J}=8.7$ and $2.2 \mathrm{~Hz}, 2 \mathrm{H}), 7.00(\mathrm{~d}, \mathrm{~J}=2.2 \mathrm{~Hz}$, $2 \mathrm{H}) ;{ }^{13} \mathrm{C} \mathrm{NMR}\left(\mathrm{CDCl}_{3}, 62.5 \mathrm{MHz}\right) \delta(\mathrm{ppm}) 175.5,161.5,160.2,160.0,140.5,140.4,137.4,130.7$, 130.2, 129.6, 129.5, 120.3, 118.7, 116.3, 107.3; MS (CI) calcd. for $\mathrm{C}_{37} \mathrm{H}_{22} \mathrm{Cl}_{2} \mathrm{O}_{8} \mathrm{~S}_{2}: m / z$ 728.01, found $729[\mathrm{M}+\mathrm{H}]^{+}$.

Precursor 16. Yield 13.1\%; m.p. $233{ }^{\circ} \mathrm{C} ;{ }^{1} \mathrm{H}$ NMR $\left(\mathrm{CDCl}_{3} / \mathrm{TFA}, 250 \mathrm{MHz}\right) \delta(\mathrm{ppm}) 7.93(\mathrm{~d}, \mathrm{~J}=9.0$ $\mathrm{Hz}, 4 \mathrm{H}), 7.92(\mathrm{~d}, \mathrm{~J}=9.0 \mathrm{~Hz}, 4 \mathrm{H}), 7.90(\mathrm{~d}, \mathrm{~J}=8.8 \mathrm{~Hz}, 4 \mathrm{H}), 7.63(\mathrm{~d}, \mathrm{~J}=8.7 \mathrm{~Hz}, 4 \mathrm{H}+4 \mathrm{H}), 7.53(\mathrm{~d}, \mathrm{~J}=$ $8.8 \mathrm{~Hz}, 4 \mathrm{H}), 7.16(\mathrm{~d}, \mathrm{~J}=8.7 \mathrm{~Hz}, 4 \mathrm{H}), 7.15(\mathrm{~d}, \mathrm{~J}=8.7 \mathrm{~Hz}, 4 \mathrm{H}) 7.13(\mathrm{~d}, \mathrm{~J}=9.0 \mathrm{~Hz}, 4 \mathrm{H}), 7.12(\mathrm{~d}, \mathrm{~J}=9.0$ $\mathrm{Hz}, 4 \mathrm{H}) ;{ }^{13} \mathrm{C} \mathrm{NMR}\left(\mathrm{CDCl}_{3}, 75 \mathrm{MHz}\right) \delta(\mathrm{ppm}) 162.1,161.8,154.7,154.5,140.6,139.7,137.1,137.0$, $135.8,134.9,130.2,130.0,129.8,129.6,128.9,128.7,120.7,119.6,119.5,117.9 ;$ MS (FAB) calcd. for $\mathrm{C}_{60} \mathrm{H}_{40} \mathrm{Cl}_{2} \mathrm{O}_{10} \mathrm{~S}_{3}: m / z$ 1086.12, found $1087[\mathrm{M}+\mathrm{H}]^{+}$.

\section{CHARACTERIZATION OF MACROCYLIC PRODUCTS}

Macrocycle 17. Yield 3.9\%; m.p. $>550{ }^{\circ} \mathrm{C} ;{ }^{1} \mathrm{H}$ NMR $\left(\mathrm{CDCl}_{3} / \mathrm{TFA}, 300 \mathrm{MHz}\right) \delta(\mathrm{ppm}) 7.99(\mathrm{~d}, \mathrm{~J}=8.3$ $\mathrm{Hz}, 8 \mathrm{H}), 7.86(\mathrm{~d}, \mathrm{~J}=8.7 \mathrm{~Hz}, 8 \mathrm{H}), 7.68(\mathrm{~d}, \mathrm{~J}=8.3 \mathrm{~Hz}, 8 \mathrm{H}), 7.11(\mathrm{~d}, \mathrm{~J}=8.7 \mathrm{~Hz}, 8 \mathrm{H}) ;{ }^{13} \mathrm{C}$ NMR 
$\left(\mathrm{CDCl}_{3} / \mathrm{TFA}, 75 \mathrm{MHz}\right) \delta(\mathrm{ppm}) 160.6,144.7,139.7,135.8,129.9,128.7,120.3$; MS (FAB) calcd. for $\mathrm{C}_{48} \mathrm{H}_{32} \mathrm{O}_{10} \mathrm{~S}_{4}: m / z$ 896.09, found $898[\mathrm{M}+\mathrm{H}]^{+}$; Anal. Calcd. for $\mathrm{C}_{48} \mathrm{H}_{32} \mathrm{O}_{10} \mathrm{~S}_{4}: \mathrm{C}$ 64.27, H 3.60. Found C 64.32, H 3.79\%.

Macrocycle 18. Yield 6.2\%; m.p. > $550{ }^{\circ} \mathrm{C} ;{ }^{1} \mathrm{H}$ NMR $\left(\mathrm{CDCl}_{3} / \mathrm{TFA}, 250 \mathrm{MHz}\right) \delta(\mathrm{ppm}) 8.03(\mathrm{~d}, \mathrm{~J}=$ $8.6 \mathrm{~Hz}, 8 \mathrm{H}), 7.97(\mathrm{~d}, \mathrm{~J}=9.0 \mathrm{~Hz}, 8 \mathrm{H}), 7.72(\mathrm{~d}, \mathrm{~J}=8.6 \mathrm{~Hz}, 4 \mathrm{H}), 7.13(\mathrm{~s}, 8 \mathrm{H}), 7.10(\mathrm{~d}, \mathrm{~J}=9.0 \mathrm{~Hz}, 8 \mathrm{H})$; ${ }^{13} \mathrm{C} \mathrm{NMR}\left(\mathrm{CDCl}_{3} / \mathrm{TFA}, 62.5 \mathrm{MHz}\right) \delta(\mathrm{ppm}) 163.6,151.8,144.9,140.9,132.9,130.5,129.0,128.3$, 123.0, 117.9; MS (FAB) calcd. for $\mathrm{C}_{60} \mathrm{H}_{40} \mathrm{O}_{12} \mathrm{~S}_{4}: m / z$ 1080.14, found $1081[\mathrm{M}+\mathrm{H}]^{+}$. Anal. Calcd. for $\mathrm{C}_{60} \mathrm{H}_{40} \mathrm{O}_{12} \mathrm{~S}_{4}$ : C 66.65, H 3.73. Found C 66.36, H 3.71\%.

Macrocycle 19. Yield 8.0\%; m.p. $400{ }^{\circ} \mathrm{C} ;{ }^{1} \mathrm{H}$ NMR $\left(\mathrm{CDCl}_{3} / \mathrm{TFA}, 300 \mathrm{MHz}\right) \delta(\mathrm{ppm}) 7.99(\mathrm{~d}, \mathrm{~J}=8.5$ $\mathrm{Hz}, 4 \mathrm{H}), 7.83(\mathrm{~d}, \mathrm{~J}=8.5 \mathrm{~Hz}, 4 \mathrm{H}), 7.75(\mathrm{~d}, \mathrm{~J}=8.8 \mathrm{~Hz}, 4 \mathrm{H}), 7.69(\mathrm{~d}, \mathrm{~J}=8.8 \mathrm{~Hz}, 4 \mathrm{H}), 7.07$ (d, J = 8.8 Hz, 4H), $7.01(\mathrm{~d}, \mathrm{~J}=8.8 \mathrm{~Hz}, 4 \mathrm{H}) ;{ }^{13} \mathrm{C} \mathrm{NMR}\left(\mathrm{CDCl}_{3} / \mathrm{TFA}, 75 \mathrm{MHz}\right) \delta(\mathrm{ppm}) 161.3,159.8,143.2,139.9$, 138.0, 135.3, 129.9, 129.5, 128.9, 128.2, 121.5, 120.0; MS (CI) calcd. for $\mathrm{C}_{36} \mathrm{H}_{24} \mathrm{O}_{8} \mathrm{~S}_{3}: m / z$ 680.06, found 680. Anal. Calcd. for $\mathrm{C}_{36} \mathrm{H}_{24} \mathrm{O}_{8} \mathrm{~S}_{3}$ : C 63.51, H 3.55. Found C 63.40, H 3.53\%. Crystal data for 19: $\mathrm{C}_{36} \mathrm{H}_{24} \mathrm{O}_{8} \mathrm{~S}_{3} . \mathrm{CHCl}_{3}, M=800.1$, triclinic, $P \overline{1}, a=11.205(14) \AA, \quad b=12.963(14) \AA, c=13.453(14)$ $\AA, \alpha=106.622(10)^{\circ}, \beta=95.431(10)^{\circ}, \gamma=99.434(10)^{\circ}, V=1826(4) \AA^{3}, T=293 \mathrm{~K}, Z=2, D_{\mathrm{c}}=1.455 \mathrm{~g}$ $\mathrm{cm}^{-3}, \mu(\mathrm{Mo}-\mathrm{K} \alpha)=0.470 \mathrm{~mm}^{-1}, F(000)=820 ; 6484$ independent reflections, $R_{1}=0.076, w R_{2}=0.222$ for 4742 independent observed reflections $\left[2 \theta \leq 52^{\circ}, I>2 \sigma(I)\right]$.

Macrocycle 20. Yield 3.1\% yield; m.p. > $500{ }^{\circ} \mathrm{C} ;{ }^{1} \mathrm{H}$ NMR $\left(\mathrm{CDCl}_{3} / \mathrm{TFA}, 300 \mathrm{MHz}\right) \delta(\mathrm{ppm}) 8.04(\mathrm{~d}$, $\mathrm{J}=8.5 \mathrm{~Hz}, 8 \mathrm{H}), 8.00(\mathrm{~d}, \mathrm{~J}=9.0 \mathrm{~Hz}, 8 \mathrm{H}), 7.96(\mathrm{~d}, \mathrm{~J}=8.9 \mathrm{~Hz}, 8 \mathrm{H}), 7.75(\mathrm{~d}, \mathrm{~J}=8.5 \mathrm{~Hz}, 8 \mathrm{H}), 7.18(\mathrm{~d}, \mathrm{~J}=$ $8.9 \mathrm{~Hz}, 8 \mathrm{H}), 7.16(\mathrm{~d}, \mathrm{~J}=9.0 \mathrm{~Hz}, 8 \mathrm{H}) ;{ }^{13} \mathrm{C} \mathrm{NMR}\left(\mathrm{CDCl}_{3} / \mathrm{TFA}, 75 \mathrm{MHz}\right) \delta(\mathrm{ppm}) 161.1,160.5,145.1$, 140.4, 135.9, 135.2, 130.6, 130.4, 129.0, 128.4, 120.6, 120.0; MS (MALDI-TOF) calcd. for $\left[\mathrm{C}_{72} \mathrm{H}_{48} \mathrm{O}_{16} \mathrm{~S}_{6}+\mathrm{Na}\right]^{+}: \mathrm{m} / z$ 1383.11, found 1383. Anal. Calcd. for $\mathrm{C}_{72} \mathrm{H}_{48} \mathrm{O}_{16} \mathrm{~S}_{6} \mathrm{C}$ 63.51, H 3.55. Found C $63.72, \mathrm{H} 3.76 \%$. 
Macrocycle 21. Yield 26.7\%; m.p. $410{ }^{\circ} \mathrm{C} ;{ }^{1} \mathrm{H}$ NMR $\left(\mathrm{CDCl}_{3} / \mathrm{TFA}, 300 \mathrm{MHz}\right) \delta$ (ppm) $7.84(\mathrm{~d}, \mathrm{~J}=8.6$ $\mathrm{Hz}, 4 \mathrm{H}), 7.69$ (d, J =8.6 Hz, 4H), $7.53(\mathrm{~d}, \mathrm{~J}=8.8 \mathrm{~Hz}, 4 \mathrm{H}), 7.41(\mathrm{t}, \mathrm{J}=8.2 \mathrm{~Hz}, 1 \mathrm{H}), 6.99$ (dd, J =8.2, 2.1 $\mathrm{Hz}, 2 \mathrm{H}), 6.81(\mathrm{~d}, \mathrm{~J}=8.8 \mathrm{~Hz}, 4 \mathrm{H}), 6.12(\mathrm{t}, \mathrm{J}=2.1 \mathrm{~Hz}, 1 \mathrm{H}) ;{ }^{13} \mathrm{C} \mathrm{NMR}\left(\mathrm{CDCl}_{3} / \mathrm{TFA}, 75 \mathrm{MHz}\right) \delta(\mathrm{ppm})$ $160.90,155.90,142.36,140.55,136.78,131.61,129.28,128.44,128.17,118.61,117.79,110.43 ;$ MS (EI) calcd.for $\mathrm{C}_{30} \mathrm{H}_{21} \mathrm{O}_{6} \mathrm{~S}_{2}: m / z$ 541.08, found 541.08. Anal.; Calcd. for $\mathrm{C}_{30} \mathrm{H}_{20} \mathrm{O}_{6} \mathrm{~S}_{2}$ : C 66.65; H 3.73; S 11.86. Found: C 66.73; H 3.72; S 11.75\%. Crystal data for $21: \mathrm{C}_{30} \mathrm{H}_{20} \mathrm{O}_{6} \mathrm{~S}_{2}, M=540.6$, monoclinic, space group $C 2 / c, a=15.4935(6) \AA, b=21.0847(6) \AA, c=10.0110(3) \AA, \beta=128.697(3)^{\circ}, V=$ 2552.39(14) $\AA^{3}, T=203 \mathrm{~K}, Z=4, D_{\mathrm{c}}=1.407 \mathrm{~g} \mathrm{~cm}^{-3}, \mu(\mathrm{Cu}-\mathrm{K} \alpha)=2.268 \mathrm{~mm}^{-1}, F(000)=1120 ; 1966$ independent reflections, $R_{1}=0.047, w R_{2}=0.126$ for 1723 independent observed reflections $[2 \theta \leq$ $\left.128^{\circ}, I>2 \sigma(I)\right]$

Macrocycle 22. Yield 14.4\%; amorphous; ${ }^{1} \mathrm{H}$ NMR $\left(\mathrm{CDCl}_{3}, 250 \mathrm{MHz}\right) \delta(\mathrm{ppm}) 7.97(\mathrm{~d}, \mathrm{~J}=8.7 \mathrm{~Hz}$, 4H), $7.92(\mathrm{~d}, \mathrm{~J}=8.4 \mathrm{~Hz}, 4 \mathrm{H}), 7.79(\mathrm{~d}, \mathrm{~J}=9.0 \mathrm{~Hz}, 4 \mathrm{H}), 7.65(\mathrm{~d}, \mathrm{~J}=8.7 \mathrm{~Hz}, 4 \mathrm{H}), 7.44(\mathrm{t}, \mathrm{J}=8.2 \mathrm{~Hz}$, 2H), $7.05(\mathrm{~d}, \mathrm{~J}=8.4 \mathrm{~Hz}, 4 \mathrm{H}), 7.03(\mathrm{dd}, \mathrm{J}=8.2$ and $2.3 \mathrm{~Hz}, 2 \mathrm{H}), 6.94(\mathrm{~d}, \mathrm{~J}=9.0 \mathrm{~Hz}, 4 \mathrm{H}), 6.85(\mathrm{dd}, \mathrm{J}=$ 8.2 and $2.3 \mathrm{~Hz}, 2 \mathrm{H}), 6.52(\mathrm{t}, \mathrm{J}=2.3 \mathrm{~Hz}, 2 \mathrm{H}) ;{ }^{13} \mathrm{C} \mathrm{NMR}\left(\mathrm{CDCl}_{3}, 62.5 \mathrm{MHz}\right) \delta(\mathrm{ppm}) 161.6,161.5$, $157.0,156.6,144.0,142.3,136.3,136.1,132.2,130.6,130.2,128.6,118.9,118.3,117.5,116.7,111.5$; MS (CI) calcd. for $\mathrm{C}_{48} \mathrm{H}_{32} \mathrm{O}_{10} \mathrm{~S}_{3}: m / z$ 864.11, found $865[\mathrm{M}+\mathrm{H}]^{+}$. Anal. Calcd. for $\mathrm{C}_{48} \mathrm{H}_{32} \mathrm{O}_{10} \mathrm{~S}_{3} \mathrm{C}$ 66.65, H 3.73. Found C 66.36, H 3.75\%.

Macrocycle 23. Yield 10.0\%; m.p. $404{ }^{\circ} \mathrm{C} ;{ }^{1} \mathrm{H}$ NMR $\left(\mathrm{CDCl}_{3} / \mathrm{TFA}, 250 \mathrm{MHz}\right) \delta(\mathrm{ppm}) 7.95(\mathrm{~d}, \mathrm{~J}=8.7$ $\mathrm{Hz}, 4 \mathrm{H}), 7.90(\mathrm{~d}, \mathrm{~J}=8.8 \mathrm{~Hz}, 4 \mathrm{H}), 7.77(\mathrm{~d}, \mathrm{~J}=8.7 \mathrm{~Hz}, 4 \mathrm{H}), 7.73(\mathrm{~d}, \mathrm{~J}=8.8 \mathrm{~Hz}, 4 \mathrm{H}), 7.61(\mathrm{~d}, \mathrm{~J}=8.9 \mathrm{~Hz}$, 4H), $7.02(\mathrm{~d}, \mathrm{~J}=8.9 \mathrm{~Hz}, 4 \mathrm{H}) ;{ }^{13} \mathrm{C} \mathrm{NMR}\left(\mathrm{CDCl}_{3} / \mathrm{TFA}, 62.5 \mathrm{MHz}\right) \delta(\mathrm{ppm}) 160.9,143.4,142.7,141.8$, 139.3, 137.8, 129.5, 129.4, 128.6, 128.5, 128.3, 121.4; MS (CI) calcd. for $\mathrm{C}_{36} \mathrm{H}_{24} \mathrm{O}_{7} \mathrm{~S}_{3} .664 .07$, found 664. Anal. Calcd. for $\mathrm{C}_{36} \mathrm{H}_{24} \mathrm{O}_{7} \mathrm{~S}_{3} .0 .1\left(\mathrm{CH}_{2} \mathrm{Cl}_{2}\right) \mathrm{C}$ 64.27, H 3.64. Found C 64.17, H 3.57. Crystal data for 23: $\mathrm{C}_{36} \mathrm{H}_{24} \mathrm{O}_{7} \mathrm{~S}_{3} \cdot \mathrm{C}_{2} \mathrm{H}_{5} \mathrm{OH}, M=722.8$, monoclinic, $P 2_{1} / \mathrm{c}, a=11.092(15) \AA, b=23.35(3) \AA, c=$ 13.887(15) $\AA, \beta=107.061(10)^{\circ}, V=3439(7) \AA^{3}, T=293(2) \mathrm{K}, Z=4, D_{\mathrm{c}}=1.396 \mathrm{~g} \mathrm{~cm}^{-3}, \mu(\mathrm{Mo}-\mathrm{K} \alpha)=$ 
$0.270 \mathrm{~mm}^{-1}, F(000)=1504 ; 5955$ independent reflections, $R_{1}=0.112, w R_{2}=0.312$ for 2680 independent observed reflections $\left[2 \theta \leq 52^{\circ}, I>2 \sigma(I)\right]$.

Macrocycle 24. Yield 13.0\%; m.p. $446{ }^{\circ} \mathrm{C} ;{ }^{1} \mathrm{H}$ NMR $\left(\mathrm{CDCl}_{3} / \mathrm{TFA}, 250 \mathrm{MHz}\right) \delta(\mathrm{ppm}) 8.32(\mathrm{~d}, \mathrm{~J}=9.0$ $\mathrm{Hz}, 2 \mathrm{H}), 8.08(\mathrm{~d}, \mathrm{~J}=8.7 \mathrm{~Hz}, 4 \mathrm{H}), 8.02(\mathrm{~d}, \mathrm{~J}=8.8 \mathrm{~Hz}, 4 \mathrm{H}), 7.95(\mathrm{~d}, \mathrm{~J}=8.7 \mathrm{~Hz}, 4 \mathrm{H}), 7.30(\mathrm{dd}, \mathrm{J}=9.0$, $2.4 \mathrm{~Hz}, 2 \mathrm{H}), 7.26(\mathrm{~d}, \mathrm{~J}=8.8 \mathrm{~Hz}, 4 \mathrm{H}), 6.19(\mathrm{~d}, \mathrm{~J}=2.4 \mathrm{~Hz}, 2 \mathrm{H}) ;{ }^{13} \mathrm{C} \mathrm{NMR}\left(\mathrm{CDCl}_{3} / \mathrm{TFA}, 62.5 \mathrm{MHz}\right) \delta$ (ppm) 164.9, 158.4, 158.3, 143.6, 140.4, 138.9, 130.5, 129.8, 129.0, 128.7, 123.5, 117.3, 116.1, 102.0; MS (CI) calcd. for $\mathrm{C}_{37} \mathrm{H}_{22} \mathrm{O}_{8} \mathrm{~S}_{2}: m / z$ 658.08, found $659[\mathrm{M}+\mathrm{H}]^{+}$. Anal. Calcd. for $\mathrm{C}_{37} \mathrm{H}_{22} \mathrm{O}_{8} \mathrm{~S}_{2} \mathrm{C} 67.47, \mathrm{H}$ 3.37. Found C 66.93, H 3.49\%. Crystal data for 24: $\mathrm{C}_{37} \mathrm{H}_{22} \mathrm{O}_{8} \mathrm{~S}_{2} \cdot 2\left(\mathrm{CHCl}_{3}\right) M=897.46, a=15.00(2) \AA$, $b=11.010(17) \AA, c=24.89(3) \AA, \beta=106.735(10)^{\circ}, V=3937(10) \AA^{3}, T=293(2) \mathrm{K}, Z=4, D_{\mathrm{c}}=1.514$ $\mathrm{g} \mathrm{cm}^{-3}, \mu(\mathrm{Mo}-\mathrm{K} \alpha)=0.595 \mathrm{~mm}^{-1}, F(000)=1824 ; 6445$ independent reflections, $R_{1}=0.086, w R_{2}=0.225$ for 1709 independent observed reflections $\left[2 \theta \leq 52^{\circ}, I>2 \sigma(I)\right]$.

Macrocycle 25. Yield 12.5\%; m.p. $484{ }^{\circ} \mathrm{C} ;{ }^{1} \mathrm{H}$ NMR $\left(\mathrm{CDCl}_{3} / \mathrm{TFA}, 250 \mathrm{MHz}\right) \delta(\mathrm{ppm}) 8.07(\mathrm{~d}, \mathrm{~J}=8.5$ $\mathrm{Hz}, 4 \mathrm{H}), 7.96(\mathrm{~d}, \mathrm{~J}=8.9 \mathrm{~Hz}, 4 \mathrm{H}), 7.90(\mathrm{~d}, \mathrm{~J}=8.9 \mathrm{~Hz}, 4 \mathrm{H}), 7.76(\mathrm{~d}, \mathrm{~J}=8.5 \mathrm{~Hz}, 4 \mathrm{H}), 7.60(\mathrm{~d}, \mathrm{~J}=8.6 \mathrm{~Hz}$, 4H), $7.59(\mathrm{~d}, \mathrm{~J}=8.6 \mathrm{~Hz}, 4 \mathrm{H}), 7.16(\mathrm{~d}, \mathrm{~J}=8.9 \mathrm{~Hz}, 4 \mathrm{H}), 7.14(\mathrm{~d}, \mathrm{~J}=8.6 \mathrm{~Hz}, 4 \mathrm{H}+4 \mathrm{H}), 7.11(\mathrm{~d}, \mathrm{~J}=8.9$ $\mathrm{Hz}, 4 \mathrm{H}) ;{ }^{13} \mathrm{C} \mathrm{NMR}\left(\mathrm{CDCl}_{3} / \mathrm{TFA}, 62.5 \mathrm{MHz}\right) \delta(\mathrm{ppm}) 163.3,163.2,154.8,154.7,144.8,140.9,137.6$, 134.0, 133.4, 130.3, 130.0, 129.0, 128.9, 128.6, 121.3, 121.1, 118.8, 118.5; MS (MALDI-TOF) calcd. for $\left[\mathrm{C}_{60} \mathrm{H}_{40} \mathrm{O}_{10} \mathrm{~S}_{3}+\mathrm{Na}\right]^{+}: \mathrm{m} / z, 1040.14$, found 1040 .

Macrocycle 26. Yield 6.6\%; m.p. $452{ }^{\circ} \mathrm{C} ;{ }^{1} \mathrm{H} \mathrm{NMR}\left(\mathrm{CDCl}_{3} / \mathrm{TFA}, 250 \mathrm{MHz}\right) \delta(\mathrm{ppm}) 8.05(\mathrm{~d}, \mathrm{~J}=8.4$ $\mathrm{Hz}, 8 \mathrm{H}), 7.95(\mathrm{~d}, \mathrm{~J}=8.9 \mathrm{~Hz}, 8 \mathrm{H}), 7.90(\mathrm{~d}, \mathrm{~J}=8.9 \mathrm{~Hz}, 8 \mathrm{H}), 7.74(\mathrm{~d}, \mathrm{~J}=8.4 \mathrm{~Hz}, 8 \mathrm{H}), 7.62(\mathrm{~d}, \mathrm{~J}=8.5 \mathrm{~Hz}$, $8 \mathrm{H}+8 \mathrm{H}), 7.15(\mathrm{~d}, \mathrm{~J}=8.9 \mathrm{~Hz}, 8 \mathrm{H}), 7.14(\mathrm{~d}, \mathrm{~J}=8.5 \mathrm{~Hz}, 8 \mathrm{H}+8 \mathrm{H}), 7.12(\mathrm{~d}, \mathrm{~J}=8.9 \mathrm{~Hz}, 8 \mathrm{H}) ;{ }^{13} \mathrm{C} \mathrm{NMR}$ $\left(\mathrm{CDCl}_{3} / \mathrm{TFA}, 62.5 \mathrm{MHz}\right) \delta(\mathrm{ppm}) 163.4,154.6,154.5,144.8,141.0,137.9,137.7,134.0,133.3,130.4$, 130.1, 129.2, 129.0, 128.5, 121.3, 118.4, 118.3; MS (MALDI) calcd. for $\left[\mathrm{C}_{120} \mathrm{H}_{80} \mathrm{O}_{20} \mathrm{~S}_{6}+\mathrm{Na}\right]^{+}: \mathrm{m} / z$ 2056.35, found 2057. Anal. Calcd. for $\mathrm{C}_{60} \mathrm{H}_{40} \mathrm{O}_{10} \mathrm{~S}_{3} \mathrm{C}$ 70.85, H 3.96. Found C 70.62, H 3.95\%. 


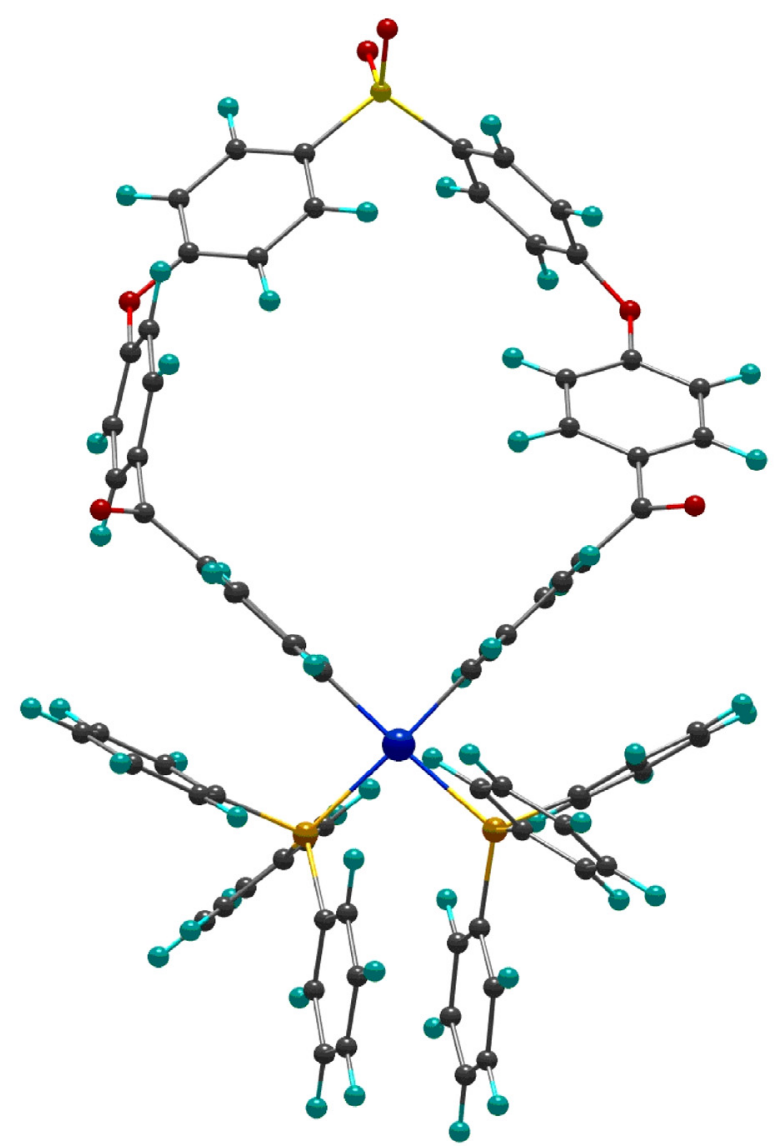

A

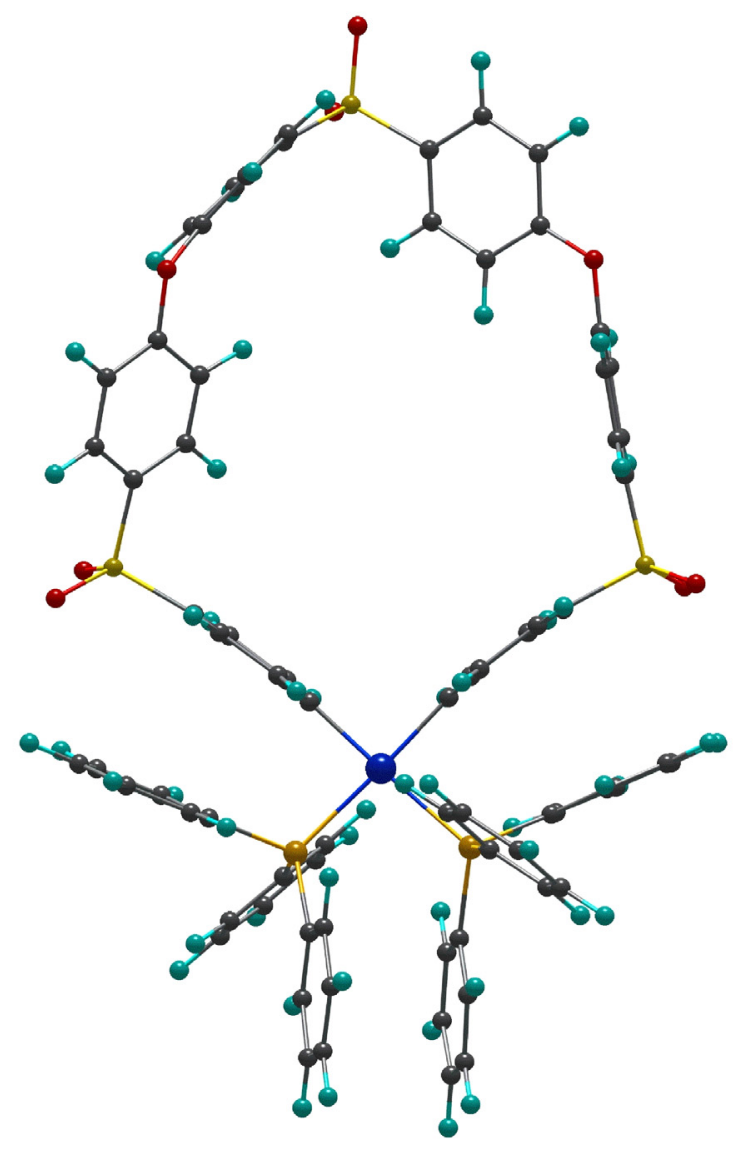

B

Figure S1. Energy-minimized models (re-optimised Dreiding-II forcefield) for cyclic organo-nickel intermediates ( $\mathbf{A}$ and $\mathbf{B}$ ) in cyclisation of the ketone- and sulfone-based precursors $\mathbf{6}$ and $\mathbf{1 1}$ respectively (c.f. Scheme 2 in paper). Note the considerable strain-induced distortions in model $\mathbf{B}$, imposed by the narrower bond angle at sulfone $\left(105^{\circ}\right)$ relative to ketone $\left(121^{\circ}\right)$. This level of strain could account, in part, for the much lower yields of macrocyclic disulfones generally obtained in nickel-catalyzed coupling reactions. 
THERMAL ELLIPSOID PLOTS (AT 50\% PROBABILITY)

\section{Compound 1}

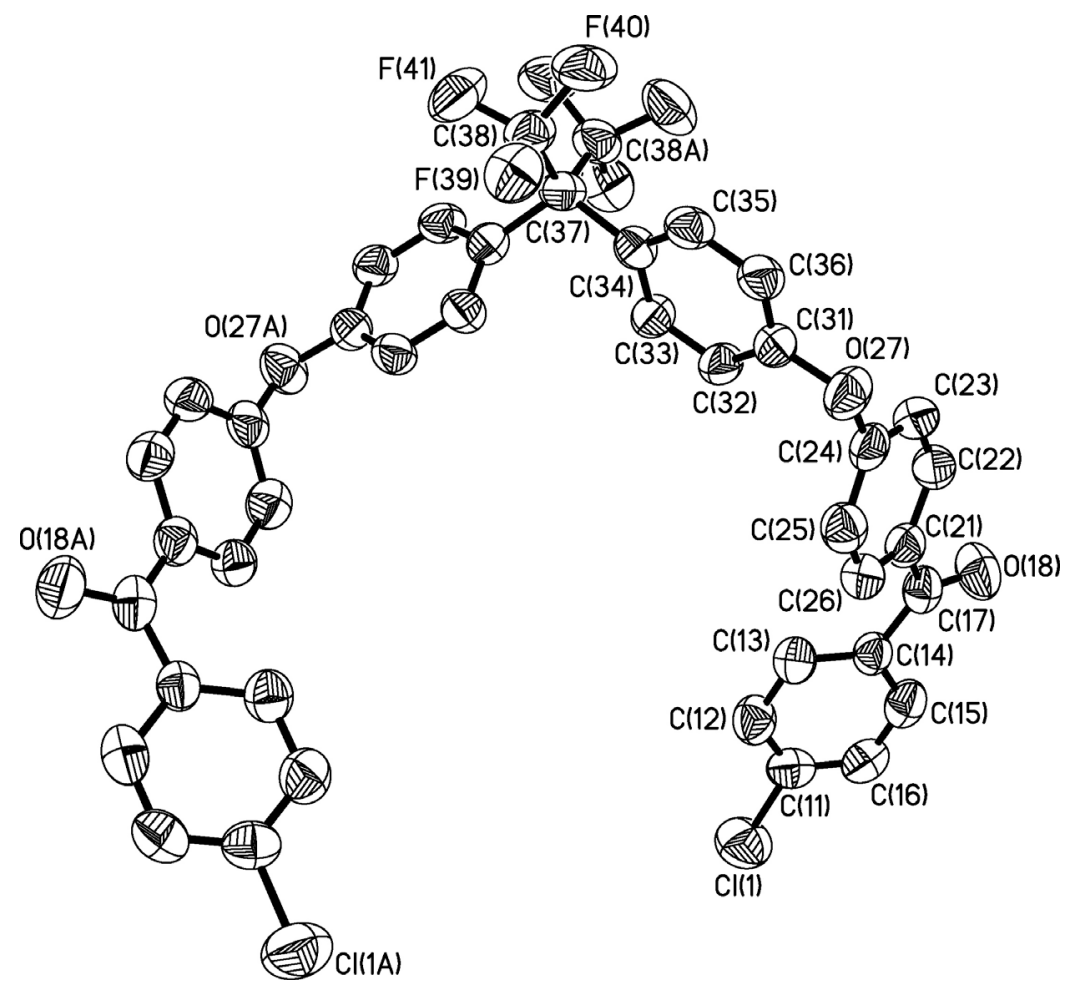

\section{Compound 6}

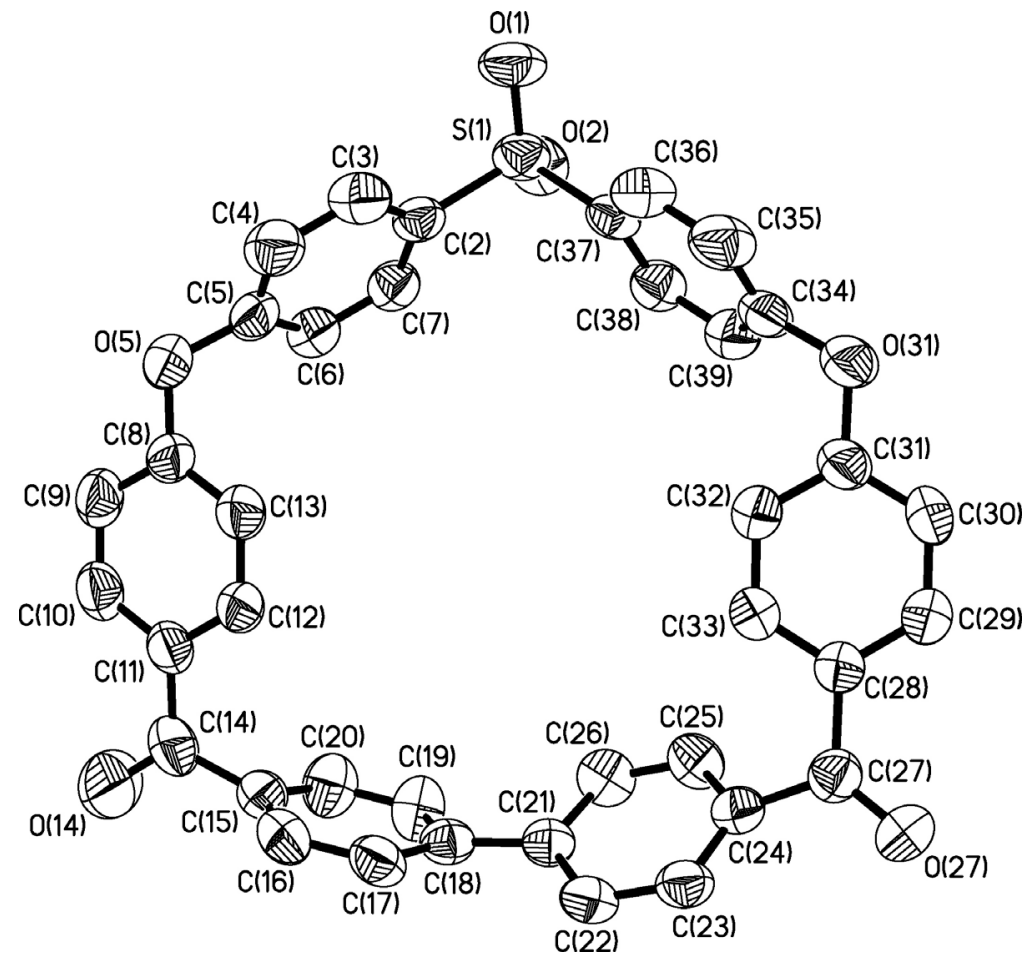




\section{Compound 8 (molecule 1)}

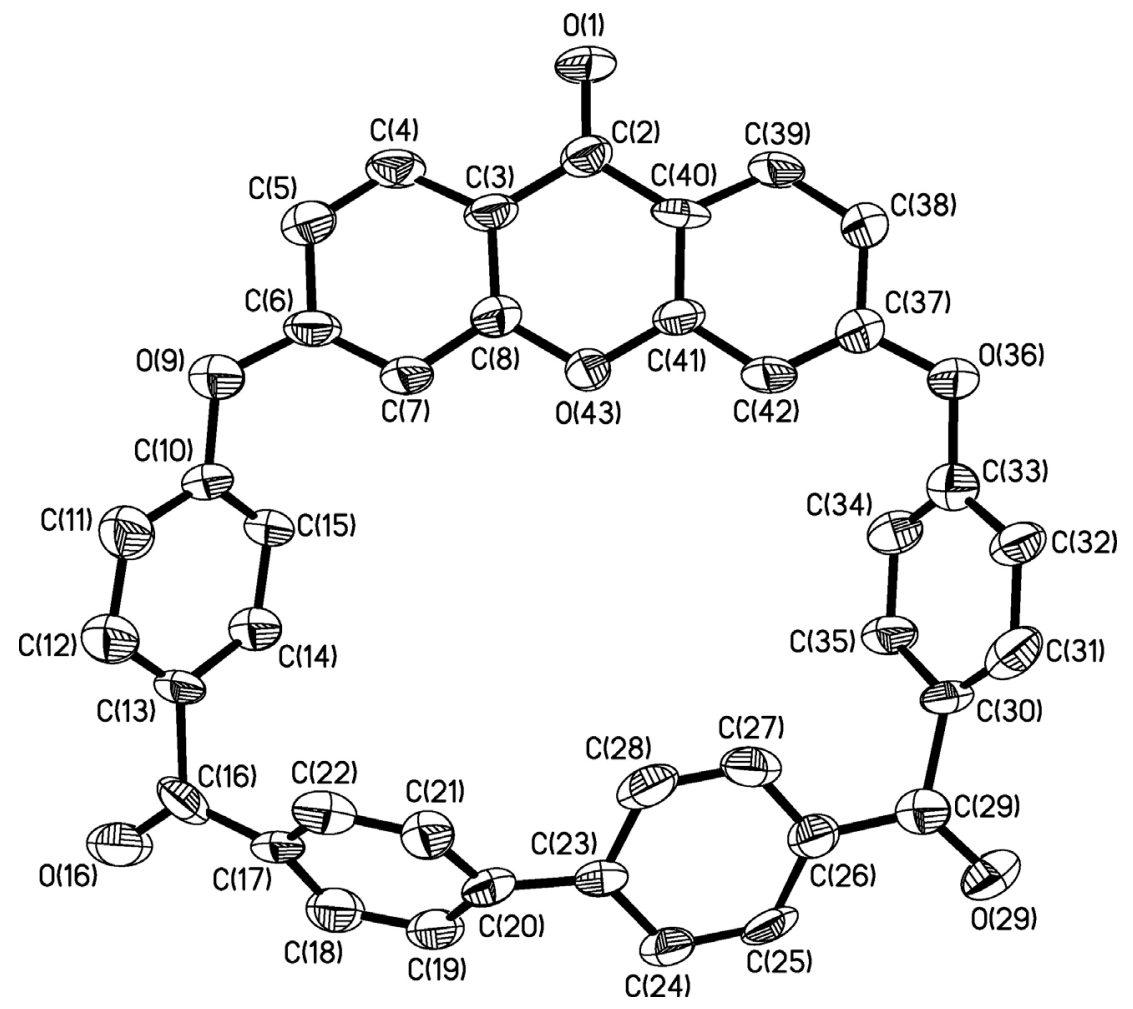

\section{Compound 8 (molecule 2)}

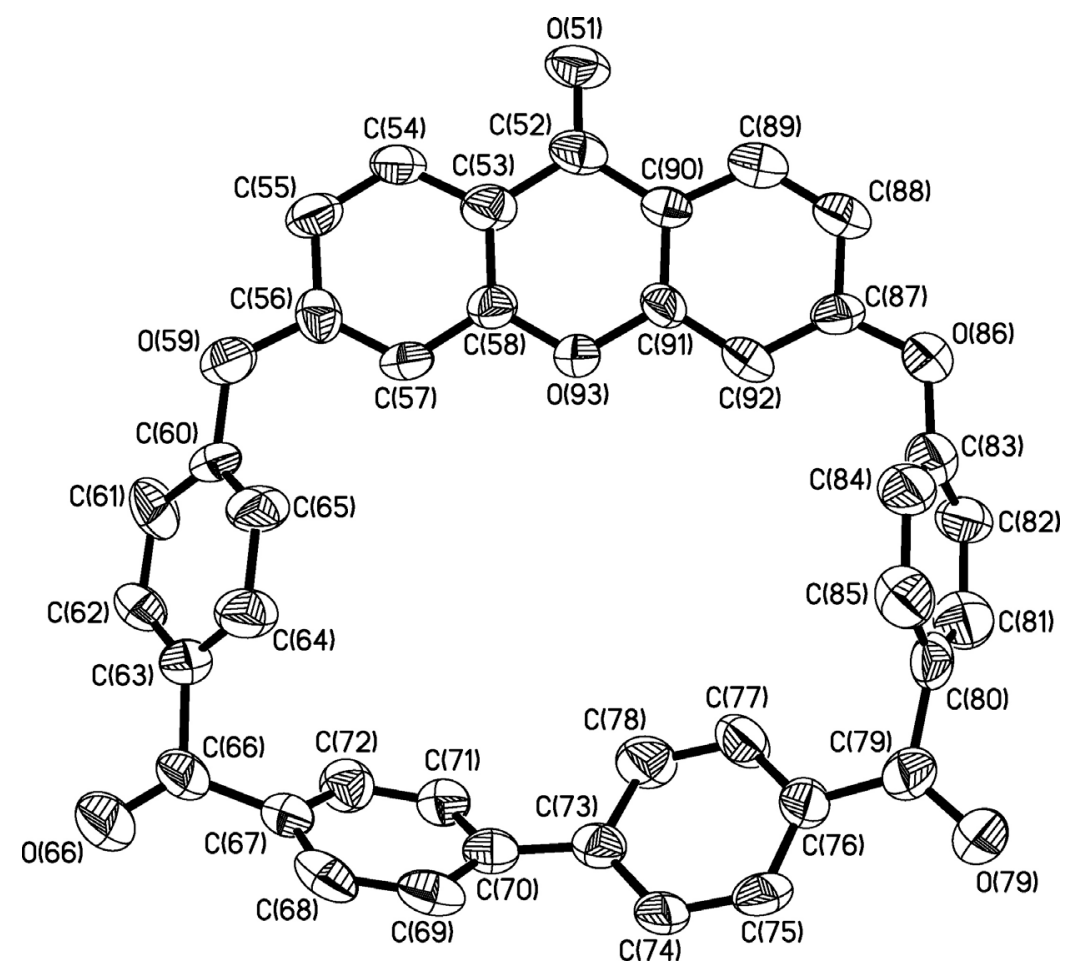




\section{Compound 19}

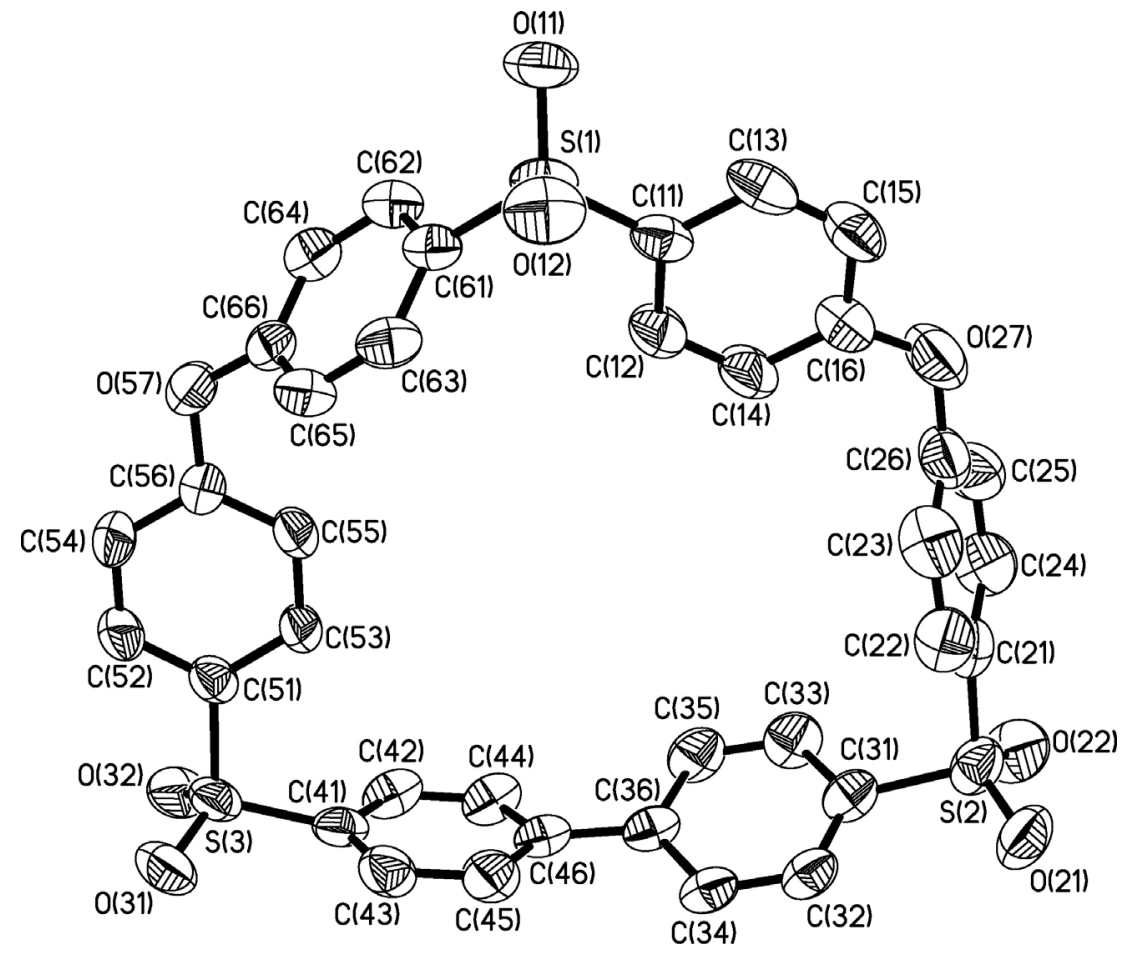

\section{Compound 23}

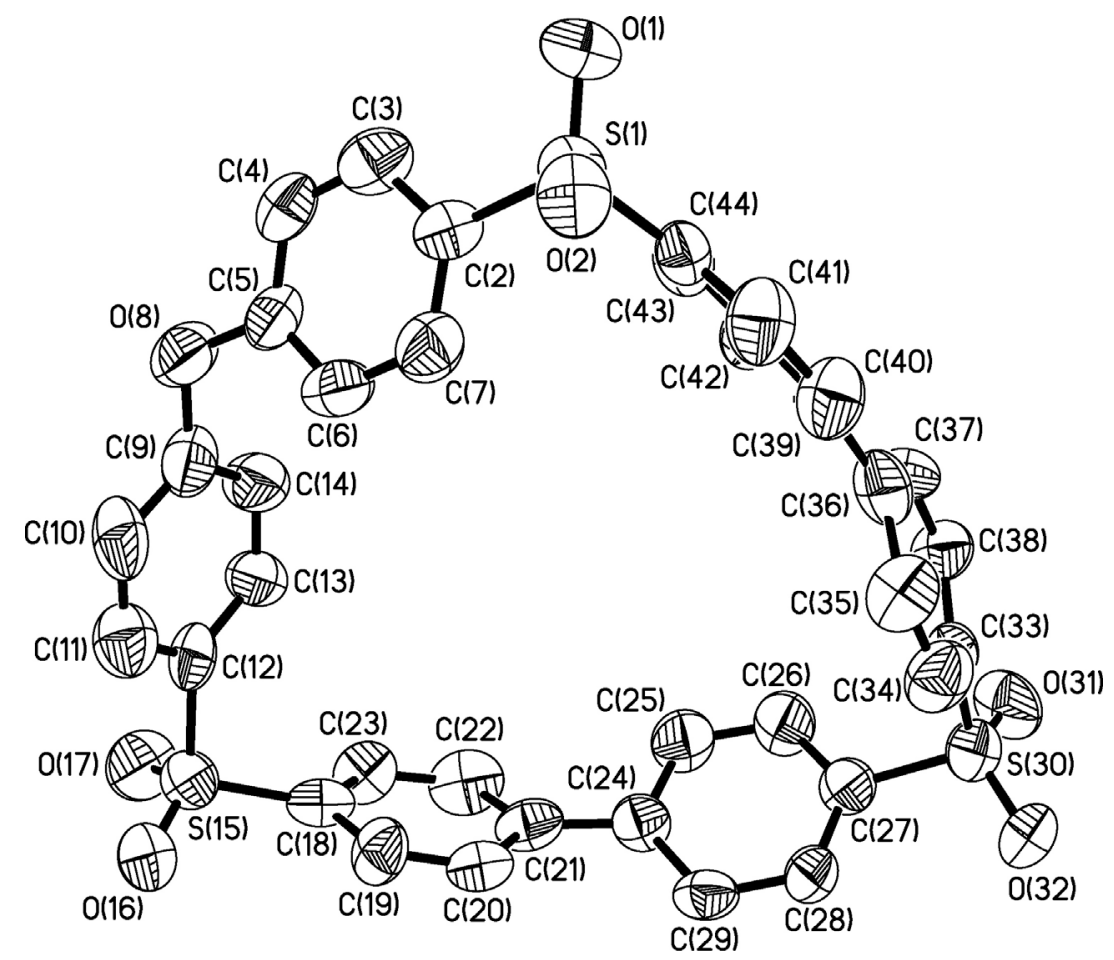




\section{Compound 24}

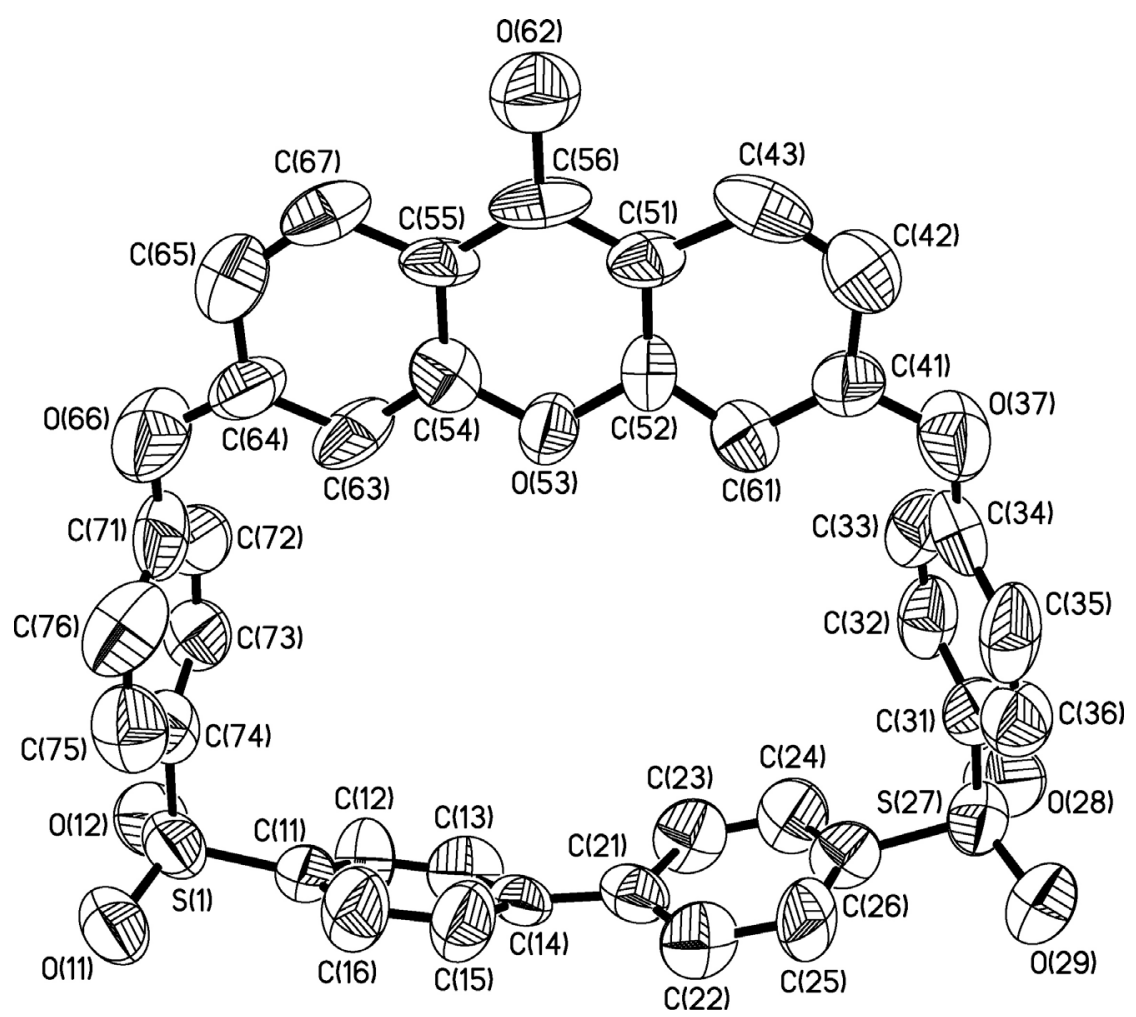

NOTE: Crystallographic data for compounds 5, 7, and 21 were deposited at the Cambridge Crystallographic Data Centre at the time of their publication in preliminary communications, and have the CCDC refcodes; RUYYAL, KEGCAA and RUYXIS respectively. 